\title{
5. Imaging techniques in diagnostic approaches
}

\author{
Claudio G De Angelis ${ }^{1}$ and Flavio Crippa ${ }^{2}$ \\ 'GastroHepatology Department, San Giovanni Battista "Molinette" Hospital, University of Turin, Turin; \\ ${ }^{2}$ SS PET, Fondazione IRCCS Istituto Nazionale dei Tumori di Milano, Milan, Italy
}

\begin{abstract}
Despite the considerable technological advances in imaging modalities which have occurred over the last years, EUS remains one of the most reliable and accurate technique for the study of gastroenteropancreatic neuroendocrine tumors. More specifically, EUS can detect very small lesions, assess the local extent and lymph node involvement and biopsy the lesion for cytophatological confirmation (EUS-FNA). In addition, nuclear medicine imaging has a relevant role in the evaluation of NET. However, its performance depends on series of patient-specific features (lesion size and uptake, depth and other anatomic features; metabolic activity, receptor expression, affinity and vacancy, tissue specificity) and technical features (choice of tracer, administered dose, and physical half-life; instrument sensitivity, acquisition technique, reader experience). In particular, current data show that PET/CT has greater intrinsic resolution and sensitivity than SPECT or SPECT/CT images resulting in improved tumor detection. However, the PET tracer of choice has not yet been identified. 18FFDG has proved to be useful as indicator of tumor aggressiveness rather than detection of extent of disease, and 68Ga-DOTA-TOC has demonstrated good results in clinical trials. 11C-5HTP has performed well in limited trials, but the 20-min half life of $11 \mathrm{C}$ precludes widespread availability. Better information concerning biodistribution and further comparative data of these agent in larger clinical trials are warranted. Free full text available at www.tumorionline.it
\end{abstract}

\section{Endoscopic ultrasound and complex diagnostics}

Gastroenteropancreatic neuroendocrine tumors (GEP NETs) have always represented a demanding challenge for diagnostic imaging techniques. GEP NETs, suspected on clinical basis, are often difficult to localize because they tend to present as very small size tumors (insulinomas, gastrinomas and carcinoids of the gastrointestinal wall) and may be multifocal, particularly when associated with multiple endocrine neoplasia type 1 (MEN1) or other inherited syndromes. Thus, $80 \%$ of the rectal lesions are less than $1 \mathrm{~cm}$ and only $5 \%$ more than $2 \mathrm{~cm}$ in diameter. Also pancreatic neuroendocrine tumors (PETs) still pose different problems in term of diagnosis, localization and therapy because of their small size, deep location in the abdomen and extrapancreatic or multiple localizations. Because these tumors are slow-growing, their prognosis is good and many patients can be cured with surgical resection. However, the majority of GEP NETs remain asymptomatic for many years and are incidentally detected on abdominal cross-sectional imaging, or become symptomatic only when disease has spread. Furthermore, even when the diagnosis is confirmed and curative surgery is considered, at least in patients without metastases, a correct pre-operative localization and staging of tumor lesions are mandatory in order to optimize surgical treatment, reduce the times and complexity of the surgical intervention and improve outcomes and results. Over the last years, we have witnessed considerable biotechnological improvements which have led to the development of increasingly complex laboratory, radiological and nuclear imaging techniques for preoperative diagnosis, detection and staging of GEP NETs. The diagnostic modalities available today range from traditional imaging methods such as trans-abdominal ul- 
trasound (US), computed tomography (CT), magnetic resonance imaging (MRI) with all their most recent applications, to more complex techniques like somatostatin receptor scintigraphy (SRS), selective intra-arterial injection of secretin or calcium (SAIS/SAIC) during angiography with hepatic venous sampling, positron emission tomography (PET), the integrated PET/CT approach and tumor markers. In this complex diagnostic scenario, endoscopic ultrasound (EUS) still plays a relevant role as a reliable and accurate technique in detecting, diagnosing, staging, following-up and also treating GEP-NETs. This paper analyzes the role of EUS in the current diagnostic armamentarium for GEP-NETs.

\subsection{Endoscopic ultrasound (EUS)}

Endoscopic ultrasound (EUS) has been one the most important advances in gastrointestinal endoscopy occurred over the last 25 years. Introduced in the early 80 's, this technique provides high-resolution images of structures not only of the mucosal surface, but also within and beyond the wall of the gastrointestinal tract, allowing the detection of lesions down to $0.3-0.5 \mathrm{~cm}$. Some practical advantages of EUS are that it is safe and minimally invasive and doesn't require general anaesthesia or hospitalization, while its major drawback is the high degree of operator dependency. During the 90's EUS has imposed itself as the most accurate and effective modality for pancreatic imaging and has become a routine procedure in the diagnostic work-up of neuroendocrine tumors.

\subsection{EUS and pancreatic endocrine tumors (PETs)}

EUS provides high resolution images of the main pancreatic duct and of the surrounding parenchyma allowing to detect structures as small as $2-3 \mathrm{~mm}$ due to the small distance between the transducer and the gland, which enables the use of higher frequency probes, from 5 to $20 \mathrm{MHz}$, with lower penetration depth but more elevated spatial resolution ${ }^{1}$. The high accuracy, reliability and positive predictive value of EUS for the preoperative detection of pancreatic endocrine tumors (PETs) were demonstrated by several authors in the 90's. In a study on 50 patients with suspected PETs we evaluated the accuracy of EUS compared to other imaging techniques in the preoperative localization, staging and following-up of tumors of the duodeno-pancreatic areas ${ }^{2}$. EUS allowed to correctly localise 20 out of the 23 pancreatic tumors, including 11 out the 12 insulinomas, three out of the eight duodenal gastrinomas and ten out of the 11 metastatic lymph nodes. Table 1 shows the detection rates obtained by EUS and other preoperative imaging modalities.

The 3 pancreatic tumors and 5 duodenal gastrinomas undetected by EUS were not localised by the other imaging techniques as well. Notably, EUS alone provided more information than the combination of all the other

\begin{tabular}{lccc}
$\begin{array}{l}\text { Table } \mathbf{1} \text { - Preoperative localization of endocrine tumors at } \\
\text { pancreatic site: comparison between EUS and other imaging } \\
\text { techniques (each patient may have multiple lesions) }\end{array}$ \\
\hline Technique & Patients (n) & \multicolumn{3}{c}{$\begin{array}{c}\text { Correct localization } \\
\text { Lesions }\end{array}$} \\
& & $20 / 23$ & 86.7 \\
\hline EUS & 19 & $4 / 23$ & 17.4 \\
US & 19 & $7 / 23$ & 30.4 \\
CT & 19 & $3 / 12$ & 25 \\
MRI & 8 & $4 / 15$ & 26.6 \\
Angiography & 11 & $2 / 13$ & 15.4 \\
SRS & 9 & &
\end{tabular}

EUS, endoscopic ultrasonography; US, ultrasonography; CT, computed tomography; MRI, magnetic resonance imaging; SRS, somatostatin-receptor scintigraphy.

imaging procedure, enabling to change clinical management in 17 out of the $39(44 \%)$ patients with PETs and to avoid surgery to 6 out of 39 patients (15.4\%). As demonstrated by previous data, EUS sensitivity was instead significantly reduced to $30 \%$ for duonenal wall gastrinomas, the detection of which can be improved by the use of intraoperative endoscopic illumination with a sensitivity of $83 \%$. Several subsequent papers demonstrated also EUS effectiveness in localising and following-up small PETs in asymptomatic patients with MEN1, and some of them recommended an early and aggressive screening of these patients using EUS, even if this datum has not been universally accepted in literature and remains controversial ${ }^{3-5}$. Over the last years the role of EUS has evolved in conjunction with the advances of other imaging techniques. So, today the detection of pancreatic insulinomas may be improved by a combined imaging protocol using both spiral multislice CT and EUS ${ }^{6}$, whereas miniprobe intraductal ultrasound (IDUS) may localize islet cell tumors undetected by endoscopic ultrasound and other techniques ${ }^{7,8}$.

\subsection{EUS with fine needle aspiration (EUS-FNA)}

Although for many years EUS has been a mere imaging modality, from the early 90's it has evolved to become also a diagnostic and therapeutic interventional technique. The introduction of EUS-guided fine needle aspiration (FNA) has enriched EUS applications with the possibility to biopsy the lesion for cytopathological confirmation. EUS with fine needle aspiration (EUSFNA) can provide a tissue diagnosis of most pancreatic masses with rather high accuracy. This is particularly useful with non-functioning neuroendocrine lesions which are otherwise difficult to diagnose preoperatively, but is important also to obtain additional histology proof of the suspected nature of the tumor even if the presence of neuroendocrine hormones has been already evidenced by biochemical tests. More specifically, a tissue diagnosis by EUS-FNA is needed when it can change patient management, i.e.: in patients with unre- 
sectable pancreatic masses or not eligible for surgery in order to guide clinician's decision making to protocols of palliative radio- o chemotherapy; when there is the justified suspicion that the pancreatic mass visualized by EUS or other imaging modalities could be a peri- or intra-pancreatic lymph node, a splenosis nodule or another type of lesion amenable of different therapeutic approaches (lymphoma, metastases, etc.); to make a differential diagnosis between benign and malignant lesion; when the patient, or sometimes the surgeon, is reluctant to perform a major surgical intervention without a cytopathological diagnosis ${ }^{2}$. Several studies have provided evidence of the high reliability of EUS-FNA in providing a tissue diagnosis of pancreatic NETs. In one study, EUS-FNA proved to be superior to computed tomography guided FNA (CT-FNA) for obtaining cells for the diagnosis of islet cell tumors of the pancreas ${ }^{9}$. Therefore, EUS-FNA can help to reduce the rate of falsepositive diagnoses resulting from peri- or intra-pancreatic lymph node or splenosis nodules and can concur to predict biological behaviour and outcome of the tumor by applying molecular biology techniques to the cell specimens obtained ${ }^{10}$. In a recent experience by our centre, EUS FNA proved to be a valuable method in the detection and diagnosis of PETs even of very small size $(<8 \mathrm{~mm})$. More specifically, all PETs distinctive cytomorphological features on FNA were shown in all adequate cases on rapid on-site evaluation; the typical cytologic findings with immunoistochemical stains allowed non only the accurate identification of PETs, but also the definition of the hormone-producing capability, biological behaviour and receptor expression ${ }^{11}$.

\subsection{Contrast-enhanced EUS (CE EUS)}

Contrast-enhanced EUS (CE EUS) is another relatively recent application of ultrasound, and can improve the already high accuracy of EUS in detecting small PETs and in posing a differential diagnosis of pancreatic lesions. A study conducted in our centre demonstrated that CE-EUS can be of help in differentiating small hypervascular pancreatic tumors, correctly identifying hypervascularity in the 2 patients with pancreatic metastases from renal cancer and hypovascularity in the pancreatic metastases from colon cancer ${ }^{12}$.

\subsection{EUS and gastrointestinal wall neuroendocrine tumors (NETS)}

EUS allows the visualization of neuroendocrine tumors (NETs), as small as $2 \mathrm{~mm}$ in diameter, located in the second and/or third layer (mucosa and/or submucosa) of the esophago-gastric, duodenal and colorectal wall. Furthermore, EUS is used for the staging of gastric and colorectal NETs by providing an accurate determination of tumor size, depth of parietal invasion and the local nodal involvement. Finally, this technique is useful in patient follow-up and in evaluating if endoscopic re- section has been radical. Thus, also for the NETs of the GI wall EUS provides valuable prognostic information and allows to plan therapeutic approach ${ }^{2}$.

As for the small intestine, which can not be reached by EUS, recent technological advances such as double or single balloon enteroscopy and video capsule seem to yield promising results, allowing to visualize and biopsy tiny NETs of the small bowel, more frequently in the ileum ${ }^{13-17}$. However, literature data on these new techniques are so far scarce, and further studies are needed.

\section{PET imaging}

Positron emission tomography (PET) is a new imaging modality which is gaining widespread use for the staging of different tumors. Based on the ability of radiolabelled tracers to be taken-up by certain tumors, it allows the study of the proliferation process through the identification of the metabolic differences between tumor cells and normal cells. Furthermore, it offers the ability to perform whole body scans. More specifically, the PET-technique can provide visualization but also the assessment of tumor biological characteristics such as substrate transport, metabolism, receptor expression and enzyme activity. According to the particular biological characteristic evaluated, PET imaging of NETs can be distinguished into non specific tumor imaging with ${ }^{18} \mathrm{~F}-\mathrm{FDG}$, substrate-based imaging with ${ }^{18} \mathrm{~F}$-FDOPA and ${ }^{11} \mathrm{C}-5 \mathrm{HTP}$, and receptor-based imaging with ${ }^{68} \mathrm{Ga}$-DOTATOC, ${ }^{68} \mathrm{Ga}$-DOTA-NOC and ${ }^{68} \mathrm{Ga}$-DOTA-TATE.

\subsection{Non specific tumor imaging: 18F-FDG PET}

PET non specific tumor imaging is based on the use of the tracer ${ }^{18} \mathrm{~F}$-labeled fluorodeoxyglucose $\left({ }^{18} \mathrm{~F}-\mathrm{FDG}\right)$ and exploits the increased glycolysis which characterizes the metabolism of tumor cells. ${ }^{18} \mathrm{~F}-\mathrm{FDG}$, a glucose analogue, was the first routinely used PET tracer in oncology and has been successfully employed for the diagnosis of cancer reflecting the increased glucose uptake in cancerous tissue. However, ${ }^{18} \mathrm{~F}-\mathrm{FDG}$-PET is currently not used for the study of neuroendocrine tumors (NETs) on a routine basis because these tumors are generally slow-growing and, accordingly, have low glycolytic activity compared to other malignancies, which may lead to a low sensitivity for ${ }^{18} \mathrm{~F}$-FDG-PET. Available data demonstrated that FDG uptake is an excellent indicator of the number of viable cancer cells, but not of their proliferative rate ${ }^{17}$. Consequently, while this tracer shows an intense uptake in the most aggressive, less differentiated neuroendocrine tumors with high proliferative activity, it usually does not show sufficient uptake in well-differentiated endocrine tumors with low proliferation rate, which represent the vast majority of NETs. However, recent data have shown that, although ${ }^{18} \mathrm{~F}-$ FDG-PET has a low diagnostic sensitivity for NETs, it 
has a strong prognostic value $\mathrm{e}^{18,19}$. T. Binderup and colleagues investigated the prognostic value of FDG uptake for predicting the survival of patients $(n=98)$ with typical and atypical NETs of gastroenteropancreatic or lung origin, compared to traditional markers for these tumors such as proliferation index Ki-67, computed tomography (CT)-verified metastases and plasma chromogranin A (CgA) levels. Results showed that patients in the FDG-positive group had an overall risk of death significantly higher as well as a progression-free survival (PFS) significantly lower compared with those in the FDG-PET-negative group, as reflected by the clear separation between Kaplan-Meier curves of survival and PFS for FDG-PET-negative patients and FDG-PET-positive patients. Similarly, the quantification of tracer accumulation, expressed as maximal standardized uptake value (SUVmax), revealed a higher risk of death for patients with a SUVmax above 9 and a lower PFS for patients with SUVmax above $3^{19}$. Furthermore, when PDF-PET was compared with traditional markers for NETs, it was found that its prognostic value exceeded that of Ki-67 index, CgA levels and the presence of metastases. Thus, although this study confirmed the overall low diagnostic sensitivity of FDP-PET for NETs, which was found to be of $58 \%$, it also demonstrated that this imaging modality was a strong independent prognostic factor for the prediction of survival both in terms of positive/negative and quantified by SUVmax ${ }^{19}$. This makes the low diagnostic sensitivity less important because a negative FDG-PET result is predictive of low aggressiveness and a high survival rate, suggesting a potential role of FDG-PET as a useful noninvasive supplement or alternative to grading based on the proliferation index as well as for detection of disease progression.

\subsection{Substrate-based imaging: 11C-5HTP and 18F-DOPA PET}

To overcome the low avidity of NETs for ${ }^{18} \mathrm{~F}-\mathrm{FDG}$ and the correlated limitations of low sensibility, other positron emitter tracers have been recently developed which are based on the extrinsic ability of these tumors to take up, accumulate and decarboxylate amine precursors. The first of this tracer, 5-hydroxytryptophan $\left({ }^{11} \mathrm{C}\right.$ 5HTP), is a direct serotonin precursor labelled with ${ }^{11} \mathrm{C}$ and has proved to be a highly more sensitive radiopharmaceutical for detection of NETs compared to ${ }^{18} \mathrm{~F}-\mathrm{FDG}$. Furthermore, whole-body ${ }^{11} \mathrm{C}-5 \mathrm{HTP}-\mathrm{PET}$ was found to be more sensitive than somatostatin receptor scintigraphy (SRS) and computed tomography (CT) in imaging small NET lesions. In a study by Orlefors and colleagues ${ }^{20}$ on 42 patients with NETs, ${ }^{11} \mathrm{C}$-5HTP-PET could image tumor lesions in $95 \%$ of subjects, provided functional information and substantially contributed to determine their nature. When ${ }^{11} \mathrm{C}-5 \mathrm{HTP}-\mathrm{PET}$ was compared with SRS and CT, in a majority of cases PET performed significantly better than the other two techniques. More specifically, ${ }^{11} \mathrm{C}$ -
5HTP-PET detected more lesions than SRS and CT in 58\% of patients and equal numbers of lesions in $34 \%$ of patients, and could visualize the primary tumor in $84 \%$ of patients compared with $47 \%$ and $42 \%$ of patients for SRS and CT, respectively. Only in 7\% of cases SRS or CT detected more lesions than PET.

Notwithstanding its high sensibility in imaging small NETs, today ${ }^{11} \mathrm{C}-5 \mathrm{HTP}$-PET can be performed only at centers with access to a cyclotron for synthesis of the radionuclide ${ }^{11} \mathrm{C}$ due to its short half-life of 20 minutes $^{20}$. This major limitation has prompted the development of a new tracer method which employs the catecholamine precursor ${ }^{18} \mathrm{~F}$-dihydroxyphenylalanin $\quad\left({ }^{18} \mathrm{~F}\right.$-DOPA), whose uptake is based on the same concept used by ${ }^{11} \mathrm{C}$ 5HTP, i.e. the property on NETs to take up amine precursors, but without the drawback associated with the rapid radioactive decay of ${ }^{11} \mathrm{C}$. Indeed, ${ }^{18} \mathrm{~F}$-DOPA PET provides high sensitivity values and enlarges the clinical accessibility of this kind of imaging method. In a prospective study by Koopmans et al. on 53 patients with metastatic carcinoid tumors, ${ }^{18} \mathrm{~F}$-DOPA PET with carbidopa pretreatment was compared with somatostatin receptor scintigraphy (SRS), computed tomography (CT) and combined SRS and $\mathrm{CT}^{5}$. Results demonstrated sensitivities of $100 \%$ for ${ }^{18} \mathrm{~F}$-DOPA PET, $92 \%$ for SRS, $87 \%$ for CT and $96 \%$ for combined SRS and CT. ${ }^{18} \mathrm{~F}-$ DOPA PET detected more lesions, more positive lesions and more lesions for region than the other imaging modalities alone or in combination ${ }^{21}$.

Koopmans and coworkers provided also a comparison of the diagnostic sensitivity of ${ }^{11} \mathrm{C}-5$-HTP and ${ }^{18} \mathrm{~F}$ DOPA PET in a large population of patients with carcinoid $(n=24)$ or pancreatic islet cell $(n=23)$ tumors $^{22}$. Both PET scans demonstrated an excellent sensitivity to detect tumor lesions. However, while in patients with carcinoid tumors ${ }^{18} \mathrm{~F}$-DOPA PET detected more lesion than ${ }^{11} \mathrm{C}-5$-HTP PET, CT and SRS, in patients with islet cells tumors ${ }^{11} \mathrm{C}$-5-HTP PET detected more tumor-positive patients and lesions than ${ }^{18} \mathrm{~F}$-DOPA PET and SRS (Table 2).

Adding CT to both PET techniques resulted on a slight further improvement in sensitivity. Therefore, ${ }^{18} \mathrm{~F}$-DOPA PET/CT and ${ }^{11} \mathrm{C}-5$-HTP PET/CT proved to be the best technique for staging of patients with carcinoid tumors and pancreatic islet cells tumors, respectively ${ }^{22}$.

\begin{tabular}{|c|c|c|c|c|}
\hline \multirow[t]{2}{*}{ Analysis } & \multicolumn{2}{|c|}{ 5-HTP sensitivity } & \multicolumn{2}{|c|}{ F-DOPA sensitivity } \\
\hline & $\begin{array}{l}\text { Carcinoid } \\
\text { tumors }\end{array}$ & $\begin{array}{c}\text { Pancreatic } \\
\text { islet cell } \\
\text { tumors }\end{array}$ & $\begin{array}{l}\text { Carcinoid } \\
\text { tumors }\end{array}$ & $\begin{array}{c}\text { Pancreatic } \\
\text { islet cell } \\
\text { tumors }\end{array}$ \\
\hline Per-patient & $100 \%$ & $100 \%$ & $96 \%$ & $89 \%$ \\
\hline Per-lesion & $89 \%$ & $96 \%$ & $98 \%$ & $80 \%$ \\
\hline
\end{tabular}




\subsection{Receptor-based imaging: 68Ga-DOTA-TOC, 68Ga-DOTA-NOC and 68Ga-DOTA-TATE PET}

The receptor-based PET imaging represents a recent technological application of the same principle underlying the use of scintigraphy with radiolabeled somatostatin analogs, i.e., the ability of neuroendocrine tumors (NETs) to overexpress somatostatin (SST) receptors. By binding to SST receptor on the tumor cell surface, SST receptor scintigraphy (SRS) has proven useful not only in diagnosing these tumors, but also in showing the content of SST receptors that might be indicative of the efficacy of treatment with otcreotide or other SST analogs. However, despite these advantages SRS has some limitations in organs with higher physiologic uptake, for example the liver, and in terms of detection of smaller lesions due to the detection limits of single photon emission computed tomography (SPECT) for radiotracers. PET technology using ${ }^{68} \mathrm{Ga}$-labelled somatostatin analogs peptide such as ${ }^{68} \mathrm{Ga}$-DOTA-TOC, ${ }^{68} \mathrm{Ga}$ DOTA-NOC and ${ }^{68} \mathrm{Ga}$-DOTA-TATE has allowed to overcome the limitations of SRS, offering improved pharmacokinetics as well as better imaging properties due to the higher spatial resolution of PET scanner. Furthermore, the synthesis of ${ }^{68} \mathrm{Ga}$ can be easily eluted from a commercially available generator, which provides the basis for a convenient and easy use of peptides labeled with this radionuclide. The advantages of PET using the new somatostatin analog ${ }^{68} \mathrm{Ga}$-labeled $1,4,7,10$ tetraazacyclododecane- $N, N, N$,' $N$ "'-tetraacetic acid-D$\mathrm{Phe}^{1}$-Tyr ${ }^{3}$-otcreotide $\left({ }^{68} \mathrm{Ga}\right.$-DOTA-TOC) compared with conventional scintigraphy and dedicated computed tomography (CT) have been clearly demonstrated ${ }^{23}$. In their study, ${ }^{68} \mathrm{Ga}$-DOTA-TOC was associated with a sensitivity of $97 \%$ compared with $52 \%$ for SPECT and $61 \%$ for CT, and in a proportion of patients provided additional information that was not obtained with the other procedures with a considerable clinical impact.

Ambrosini et al. ${ }^{24}$ demonstrated also that the use of the somatostatin analog ${ }^{68} \mathrm{Ga}$-labeled DOTA-NOC was associated with an increased sensitivity and accuracy in the detection of primary and metastatic tumors compared with ${ }^{18} \mathrm{~F}$-DOPA. In this study, which involved patients $(\mathrm{n}=13)$ with gastroenteropancreatic and lung neuroendocrine tumors, ${ }^{68} \mathrm{Ga}$-DOTA-NOC PET was positive in $13 / 13$ cases while ${ }^{18} \mathrm{~F}$-DOPA PET was positive in 9/13 cases. On a lesion basis, ${ }^{68} \mathrm{Ga}-\mathrm{DOTA}-\mathrm{NOC}$ identified more lesions than ${ }^{18} \mathrm{~F}$-DOPA (71 vs 45), especially in the liver, lung and lymph nodes. ${ }^{68} \mathrm{Ga}-$ DOTA-NOC correctly identified the primary site in six of eight non-operated cases, while ${ }^{18} \mathrm{~F}$-DOPA identified the primary only in two of eight cases ${ }^{24}$.

\section{References}

1. De Angelis C, Repici A, Carucci P, Bruno M, Goss M, Mezzabotta L, Pellicano R, Saracco G, Rizzetto M: Pancreatic cancer imaging: the new role of endoscopic ultrasound. JOP J Pancreas, 8: 85-97, 2007.

2. De Angelis C, Carucci P, Repici A, Rizzetto M: Endosonography in decision making and management of gastrointestinal endocrine tumors. Eur J Ultrasound, 10: 139-150, 1999.

3. Gauger PG, Scheiman JM, Wamsteker EJ, Richards ML, Doherty GM, Thompson NW: Role of endoscopic ultrasonography in screening and treatment of pancreatic tumors in asymptomatic patients with multiple endocrine neoplasia type 1. Br J Surg, 90: 748-754, 2003.

4. Langer P, Kann PH, Fendrich V, Richter G, Diehl S, Rothmund M, Bartsch DK: Prospective evaluation of imaging procedures fort he detection of pancreaticoduodenal endocrine tumors in patients with multiple endocrine neoplasia type 1. World J Surg, 28:1317-1322, 2004.

5. Thomas-Marques L, Murat A, Delemer B, Penfornis A, Cardot-Bauters C, Baudin E, Niccoli-Sire P, Levoir D, Choplin Had B, Chabre O, Jovenin N, Cadiot G; Groupe de Tumeurs Endocrines (GTE): Prospective endoscopic ultrasonographic evaluation of the frequency of non-functioning pancreaticoduodenal endocrine tumors in patients with multiple endocrine neoplasia type 1 . Am J Gastroenterol, 101: 266-273, 2006.

6. McLean AM, Fairclough MD: Endoscopic ultrasound in the localisation of pancreatic islet cell tumors. Best Pract Res Clin Endocrinol Metab, 19: 177-193, 2005.

7. Levy MJ, Vazquez-Sequeiros E, Wiersema MJ: Evaluation of the pancreaticobiliary ductal systems by intraductal US. Gastrointestinal Endoscopy, 55: 397-408, 2002.

8. Menzel J, Domschke W: Intraductal ultrasonography may localize islet cell tumours negative on endoscopic ultrasound. Scand J Gastroenterol, 33: 109-112, 1998.

9. Jhala D, Eloubeidi M, Chhieng DC, Frost A, Eltoum IA, Roberson J, Jhala N: Fine needle aspiration biopsy of the islet cell tumor of pancreas: a comparison between computerized axial tomography and endoscopic ultrasoundguided fine needle aspiration biopsy. Ann Diagn Pathol, 6 (2): 106-112, 2002.

10. Chatzipantelis P, Konstantinou P, Kaklamanos M, Apostolou G, Salla C: The role of cytomorphology and proliferative activity in predicting biologic behaviour of pancreatic neuroendocrine tumors: a study by EUS-FNA cytology. Cancer Cytopathol, 117: 211-216, 2009.

11. De Angelis C: EUS-guided cytologic diagnosis of pancreatic endocrine tumors (PETs): impact of on-site cytopathologic assessment. Digestive and Liver Disease, 41: 63 (abstract), 2009.

12. De Angelis C: The challenging differential diagnosis of solid pancreatic masses: is there a role for contrast-enhanced EUS (CE-EUS)? Digestive and Liver Disease; 41: 61-62 (abstract), 2009.

13. Yamaguchi T, Manabe N, Tanaka S, Fukumoto A, Shimamoto M, Nakao M, Kamino D, Chayama K: Multiple carcinoid tumors of the ileum preoperatively diagnosed by enteroscopy with the double-balloon technique. Gastrointest Endosc, 62: 315-318, 2005.

14. Scherübl H, Faiss S, Tschöpe R, Zeitz M: Double-balloon enteroscopy for the detection of midgut carcinoids. Gastrointest Endosc, 62: 994, 2005.

15. Yamagishi H, Fukui H, Shirakawa K, Oinuma T, Hiraishi H, Terano A, Fujimori T, Nakamura T: Early diagnosis and successful treatment of small-intestinal carcinoid tumor: useful combination of capsule endoscopy and double-balloon endoscopy. Endoscopy, 39: 243-244, 2007.

16. Fukumoto A, Manabe N, Tanaka S, Yamaguchi T, Matsumoto Y, Chayama K: Usefulness of EUS with double balloon enteroscopy for diagnosis of small bowel diseases. Gastrointest Endosc, 65: 412-420, 2007. 
17. Higashi K, Clavo AC, Wahl RL: Dose FDG uptake neasure proliferative activity of human cancer cells? In vitro comparison with DNA flow cytometry and tritiated thymidine uptake. J Nucl Med, 34: 414-419, 1993.

18. Kayani I, Conry BG, Groves AM, Win T, Dickson J, Caplin M, Bomanji JB: A comparison of 68Ga-DOTATATE and 18FFDG PET/CT in pulmonary neuroendocrine tumors. J Nucl Med 50: 1927-1932, 2009.

19. Binderup T, Knigge U, Loft A, Federspiel B, Kjaer A: 18FFluorodeoxyglucose positron emission tomography predicts survival pf patients with neuroendocrine tumors. Clin Cancer Res, 16: 978-985, 2010.

20. Orlefors H, Sundin A, Garske U, Juhlin C, Oberg K, Skogseid B, Langstrom B, Bergstrom M, Eriksson B: Whole-body 11C-5-hydroxytryptophan positron emission tomography as a universal imaging technique for neuroendocrine tumors: comparison with somatostatin receptor scintrigraphy and computed tomography. J Clin Endocrinol Metab, 90: 3392-3400, 2005.

21. Koopmans KP, de Vries EG, Kema IP, Elsinga PH, Neels OC, Sluiter WJ, van der Horst-Schrivers AN, Jager PL: Staging of carcinoid tumours with 18F-DOPA PET: a prospective, diagnostic accuracy study. Lancet Oncol, 7: 728-734, 2006.

22. Koopmans KP, Neels OC, Kema IP, Elsinga PH, Sluiter WJ, Vanghillewe K, Brouwers AH, Jager PL, de Vries EG: Improved staging of patients with carcinoid and islet cell tumors with 18F-dihydroxy-phenyl-alanine and 11C-5hydroxy-tryptophan positron emission tomography. J Clin Oncol, 26: 1489-1495, 2008.

23. Gabriel M, Decristoforo C, Kendler D, Dobrozemsky G, Heute D, Uprimny C, Kovacs P, Von Guggenberg E, Bale R, Virgolini IJ: 68Ga-DOTA-Tyr3-Otcreotide PET in neuroendocrine tumors: comparison with somatostatin receptor scintigraphy and CT. J Nucl Med, 48: 508-518, 2007.

24. Ambrosini V, Tomassetti P, Castellucci P, Campana D, Montini G, Ribello D, Nanni C, Rizzello A, Franchi R, Fanti S: Comparison between 68Ga-DOTA-NOC and 18F-DOPA PET for the detection of gastro-entero-pancreatic and lung neuro-endocrine tumours. Eur J Nucl Med Mol Imaging, 35: 1431-1438, 2008. 